\title{
Probing gas and dust in the tidal tail of NGC 5221 with the type la supernova iPTF16abc ${ }^{\star}$
}

\author{
R. Ferretti ${ }^{1}$, R. Amanullah ${ }^{1}$, A. Goobar ${ }^{1}$, T. Petrushevska ${ }^{1}$, S. Borthakur ${ }^{2}$, M. Bulla $^{1}$, O. Fox ${ }^{3}$, E. Freeland ${ }^{4}$, \\ C. Fremling ${ }^{4}$, L. Hangard ${ }^{1}$, and M. Hayes ${ }^{4}$ \\ 1 Department of Physics, The Oskar Klein Centre, Stockholm University, Albanova, 10692 Stockholm, Sweden \\ e-mail: raphael.ferretti@fysik.su.se \\ 2 Arizona State University, School of Earth and Space Exploration, 650 E. Tyler Mall Tempe, AZ 85281, USA \\ 3 Space Telescope Science Institute, 3700 San Martin Drive, Baltimore, MD 21218, USA \\ 4 Department of Astronomy, The Oskar Klein Center, Stockholm University, Albanova, 10691 Stockholm, Sweden
}

Received 20 June 2017 / Accepted 25 August 2017

\section{ABSTRACT}

\begin{abstract}
Context. Type Ia supernovae (SNe Ia) can be used to address numerous questions in astrophysics and cosmology. Due to their well known spectral and photometric properties, SNe Ia are well suited to study gas and dust along the lines-of-sight to the explosions. For example, narrow Na I D and Ca II H\&K absorption lines can be studied easily, because of the well-defined spectral continuum of $\mathrm{SNe}$ Ia around these features.

Aims. We aim to study the gas and dust along the line-of-sight to iPTF16abc, which occurred in an unusual location, in a tidal arm, $80 \mathrm{kpc}$ from centre of the galaxy NGC 5221.

Methods. Using a time-series of high-resolution spectra, we have examined narrow Na I D and Ca II H\&K absorption features for variations in time, which would be indicative for circumstellar (CS) matter. Furthermore, we have taken advantage of the well known photometric properties of SNe Ia to determine reddening due to dust along the line-of-sight.

Results. From the lack of variations in $\mathrm{Na}$ I D and $\mathrm{Ca}$ II H\&K, we determine that none of the detected absorption features originate from the CS medium of iPTF16abc. While the Na I D and Ca II H\&K absorption is found to be optically thick, a negligible amount of reddening points to a small column of interstellar dust.

Conclusions. We find that the gas along the line-of-sight to iPTF16abc is typical of what might be found in the interstellar medium (ISM) within a galaxy. It suggests that we are observing gas that has been tidally stripped during an interaction of NGC 5221 with one of its neighbouring galaxies in the past $\sim 10^{9} \mathrm{yr}$. In the future, the gas clouds could become the locations of star formation. On a longer time scale, the clouds might diffuse, enriching the circum-galactic medium (CGM) with metals. The gas profile along the line-of-sight should be useful for future studies of the dynamics of the galaxy group containing NGC 5221.
\end{abstract}

Key words. supernovae: individual: iPTF16abc - galaxies: individual: NGC 5221 - galaxies: ISM - galaxies: interactions

\section{Introduction}

Type Ia supernovae (SNe Ia) have such uniform properties that they are frequently described as "standard candles", which, among other applications, enables detailed measurements in cosmology (see for instance Goobar \& Leibundgut 2011). The wellknown brightness and spectral energy distribution (SED) of $\mathrm{SNe}$ Ia can also be used to study gas and dust along the linesof-sight.

Due to the well defined continuum of SNe Ia, typical absorption features of the interstellar medium (ISM) of their host galaxies, such as $\mathrm{Na}$ I D and $\mathrm{Ca}$ II $\mathrm{H} \& \mathrm{~K}$, can frequently be identified. Furthermore, diffuse interstellar bands (DIBs), which are largely of unknown chemical origin (Snow 2001; Snow \& McCall 2006), are sometimes detected in the spectra (e.g. Sollerman et al. 2005). One can also take advantage of the standard candle properties of SNe Ia to study dust in the ISM. Comparing the lightcurves of a given SN Ia with an

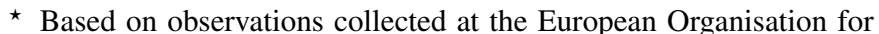
Astronomical Research in the Southern Hemisphere under ESO DDT programme 297.D-5005(A), P. I. Ferretti.
}

SED template, extinction and reddening can be determined to study the dust properties along the line-of-sight.

For example, the nearest SN Ia in modern times, SN 2014J, (Goobar et al. 2014), has been used to study details of the ISM composition and structure of M82 (Welty et al. 2014; Ritchey et al. 2015; Yang et al. 2017). Interestingly, an unusual extinction curve was identified which possibly points to very small dust grains in the ISM or dust in the circumstellar (CS) medium of the supernova (Amanullah et al. 2014; Foley et al. 2014).

SNe Ia can be used to study a large variety of lines-of-sight. Due to the large delay times of $\approx 10^{8}-10^{9} \mathrm{yr}$ between the formation of a SN Ia progenitor system and the explosion (Maoz et al. 2012), they are known to occur almost anywhere in their host galaxies. An extreme example is PTF10ops, which was located at a projected distance of $148 \mathrm{kpc}$ from the nearest possible host galaxy (Maguire et al. 2011).

It is thus possible for $\mathrm{SNe}$ Ia to occur in regions with low stellar density, such as tidally stripped spiral arms of galaxies. Tidal arms are thought to be the result of close interactions of neighbouring galaxies (as shown in the simulations by Toomre \& Toomre 1972). SNe Ia occurring in such locations can 


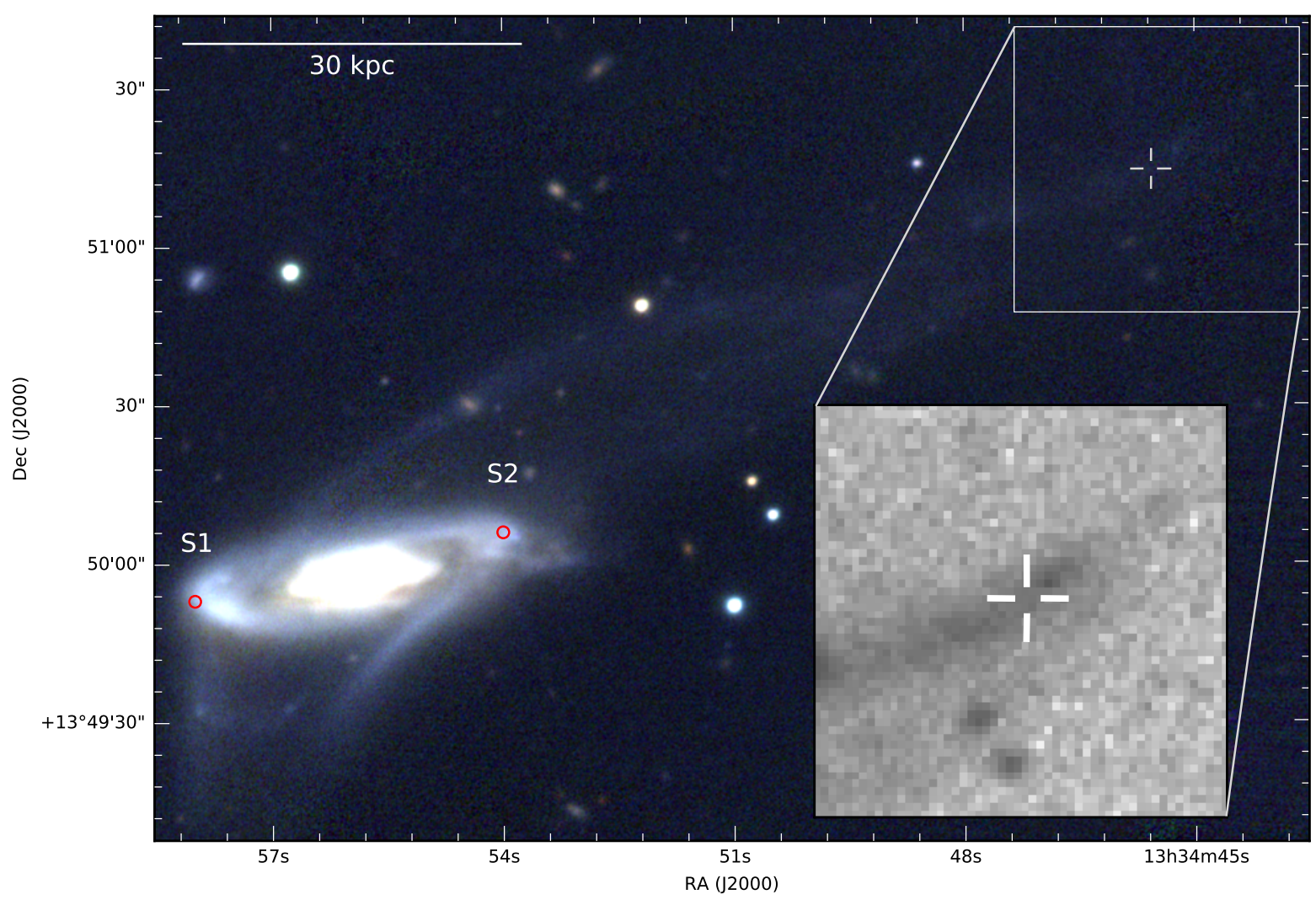

Fig. 1. Location of iPTF16abc, marked in the inset, with respect to its host galaxy NGC 5221. The tidal tail is visible spanning the space between the galaxy and the supernova location. Coordinates at which SDSS spectra of NGC 5221 are available are marked and labelled S1 and S2. The main image is a colour composite of the publicly available PanSTARRS data (Flewelling et al. 2016). The inset is a deep stack of pre-explosion iPTF P48 R-band images.

be used to study the interstellar gas and dust properties of regions that have been affected by gravitational interactions. The ISM in tidal arms can be the location of future star formation (Schombert et al. 1990) and enrich the circumgalactic medium (CGM) with metals.

To date no SN Ia located in a tidal arm has been studied in detail, although at least one case is known (SN 2013de, Drake et al. 2013a,b). Here we have investigated iPTF16abc, a SN Ia which occurred in the tidal arm of its host galaxy, NGC 5221. Due to the large displacement of iPTF16abc from its host, the supernova was a good candidate to search for the presence of CS gas via photoionisation using the method described in Ferretti et al. (2016). Furthermore, the obtained high-resolution spectra and photometry could be used to study the spiral arm where the supernova is located.

In the following, we present the discovery and observations of iPTF16abc (Sect. 2). We then describe the narrow absorption features detected in high-resolution spectra, as well as the photometric reddening of iPTF16abc (Sect. 3). We further show that the deep absorption features cannot be due to CS gas surrounding the supernova, but are part of the tidally stripped ISM of NGC 5221 (Sect. 4). Finally, we compare the line-of-sight with NGC 5221 and discuss the features in the context of the galaxy group in which NGC 5221 is located (Sects. 5 and 6).

\section{Discovery and observations of iPTF16abc}

The intermediate Palomar Transient Factory (iPTF) first reported the discovery of iPTF16abc (Miller et al. 2016), located $170^{\prime \prime}$ from the galaxy NGC 5221 at RA,
Dec $=13: 34: 45.492+13: 51: 14.30(J 2000)$, a line-of-sight that has low Milky Way reddening with $E(B-V)_{\mathrm{MW}}=0.028 \mathrm{mag}$ (Schlafly \& Finkbeiner 2011). A colour composite image of NGC 5221 and the location of iPTF16abc is shown in Fig. 1. The supernova was discovered on 4.4 April 2016, while the first detection was on 3.4 April and the last non-detection occurred on 2.4 April (UT dates are used throughout the paper).

A classification spectrum of iPTF16abc was obtained with the DeVeny spectrograph on the Discovery Channel Telescope (DCT; Cenko et al. 2016) on 5.3 April, which determined it to be a SN Ia, possibly belonging to the subclasses resembling SNe 1991T or 1999aa. A spectrum obtained with Gemini $\mathrm{N}+\mathrm{GMOS}$ on 5.4 April revealed the presence of deep Na I D and $\mathrm{Ca}$ II H\&K absorption features. In response, extensive photometric and spectroscopic follow-up were triggered (Miller et al. 2017).

As part of the follow-up of iPTF16abc, we received Director's Discretionary Time at the European Organisation for Astronomical Research in the Southern Hemisphere (ESO DDT programme 297.D-5005(A), P.I. Ferretti). The spectra taken with $\mathrm{X}$-shooter $(R \simeq 7500$, Vernet et al. 2011) and the Ultraviolet and Visual Echelle Spectrograph (UVES; $R \simeq 50000$ Dekker et al. 2000) both on Kueyen (UT2) $8.2 \mathrm{~m}$ unit at the Very Large Telescope (VLT) are summarised in Table 1. The X-shooter and UVES spectra were reduced using the REFLEX (ESOREX) reduction pipeline provided by ESO (Modigliani et al. 2010). Where necessary, telluric features were corrected for using Molecfit (Smette et al. 2015; Kausch et al. 2015).

We analysed the photometry of iPTF16abc presented in Miller et al. (2017). From the Palomar Observatory 48-inch 


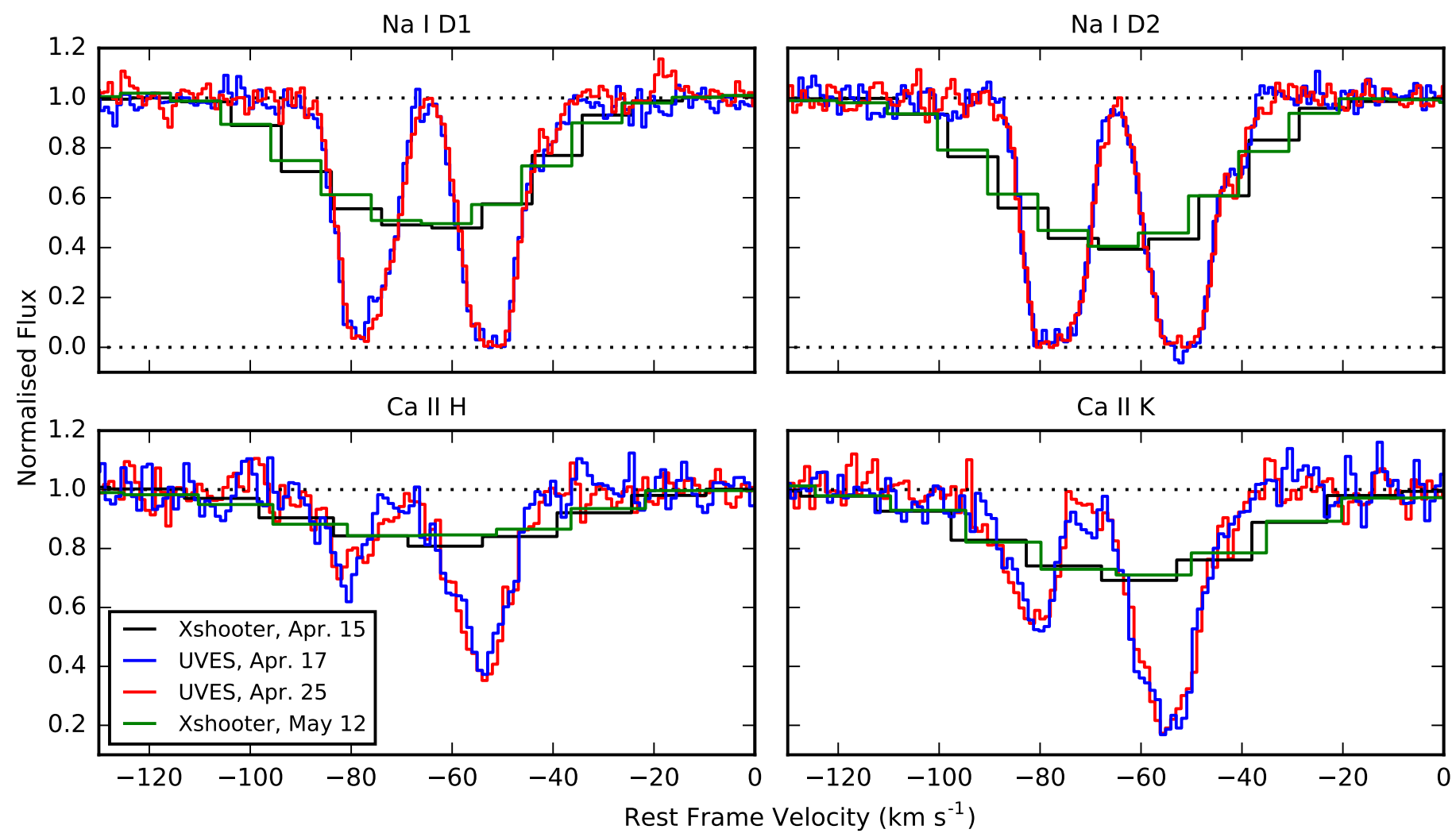

Fig. 2. Na I D doublet and Ca II H\&K of iPTF16abc by UVES and X-shooter. In the UVES spectra, two distinct groups of absorption features with similar velocities are visible, while they are unresolved with X-shooter. The substructure of these groups is difficult to further discern due to the optical thickness of the absorbers.

Table 1. Mid- and high-resolution spectra obtained with stretch-corrected phases in the rest frame with respect to $B$-band maximum.

\begin{tabular}{lccccc}
\hline \hline Instrument & MJD & UT date & $\begin{array}{c}\text { Exp. time } \\
(\mathrm{s})\end{array}$ & $\begin{array}{c}\text { Phase } \\
\text { (days) }\end{array}$ & Set-up \\
\hline X-shooter & 57492.2 & Apr. 14.2 & 1755 & -6.6 & UVB 1.0"/VIS 0.9"/NIR 0.9" \\
UVES & 57495.2 & Apr. 17.2 & $2 \times 1700$ & -3.6 & $0.8^{\prime \prime}$ DIC1 390+580 \\
UVES & 57503.1 & Apr. 25.1 & 3450 & 4.3 & $0.8^{\prime \prime}$ DIC1 390+580 \\
X-shooter & 57520.0 & May 12.0 & 1829 & 21.2 & UVB 1.0" /VIS 0.9"/NIR 0.9" \\
\hline
\end{tabular}

telescope (P48) we use $g$ - and $R$-band photometry which was obtained as part of the regular iPTF survey. We also used $g r$ and $i$-band photometry of the Palomar Observatory 60-inch telescope (P60) and BVgr- and $i$-band from the Las Cumbres Observatory (LCO). Ultraviolet (UV) photometry from the Ultraviolet/Optical Telescope (UVOT; Roming et al. 2005) on the Swift spacecraft (Gehrels et al. 2004) uvm2-filter and riZYJ- and $H$-band with the Reionisation and Transient Infrared/Optical Project (RATIR; Fox et al. 2012) was obtained and used for this analysis.

\section{Absorption features and reddening}

Deep well-defined narrow absorption lines corresponding to $\mathrm{Na}$ I D and Ca II H\&K at the rest frame of NGC $5221(z=$ 0.0234 , Courtois \& Tully 2015) are evident in all X-shooter and UVES spectra obtained. We detected two distinct groups of absorbers at different radial velocities in the UVES spectra. The absorption line profiles can be seen in Fig. 2, where the two deep feature groups are centred at a radial velocity $v_{r} \approx-77$ and $-51 \mathrm{~km} \mathrm{~s}^{-1}$, with respect to the host galaxy rest frame.
Table 2. Average Voigt profile parameters of $\mathrm{Ca}$ II $\mathrm{H} \& \mathrm{~K}$ in the two UVES spectra.

\begin{tabular}{ccc}
\hline \hline $\begin{array}{c}v \\
\left(\mathrm{~km} \mathrm{~s}^{-1}\right)\end{array}$ & $\begin{array}{c}b \\
\left(\mathrm{~km} \mathrm{~s}^{-1}\right)\end{array}$ & $\begin{array}{c}\log _{10}\left\{N_{\mathrm{Ca}}\right\} \\
\left(\mathrm{cm}^{-2}\right)\end{array}$ \\
\hline-88. & $3.1 \pm 1.3$ & $11.05 \pm 0.09$ \\
-80. & $5.0 \pm 0.4$ & $11.93 \pm 0.02$ \\
-58. & $5.0 \pm 0.3$ & $12.06 \pm 0.02$ \\
-52. & $3.6 \pm 0.3$ & $12.24 \pm 0.03$ \\
-43. & $3.1 \pm 1.1$ & $11.20 \pm 0.08$ \\
\hline
\end{tabular}

Both groups of absorption line features are visibly asymmetric, indicating that the absorption features are composed of several blended components. The $\mathrm{Na}$ I D lines are too saturated and blended to determine any further substructure with confidence. Therefore, only $\mathrm{Na}$ I column density upper limits can be determined. Using VPFIT ${ }^{1}$, we fitted Voigt profiles to $\mathrm{Ca}$ II $\mathrm{H} \& \mathrm{~K}$. In Table 2, we present the average Doppler width $(b)$ and column densities $(N)$ of a five-component fit. Since all the features

\footnotetext{
1 http://www. ast. cam.ac.uk/ rfc/vpfit.html
} 


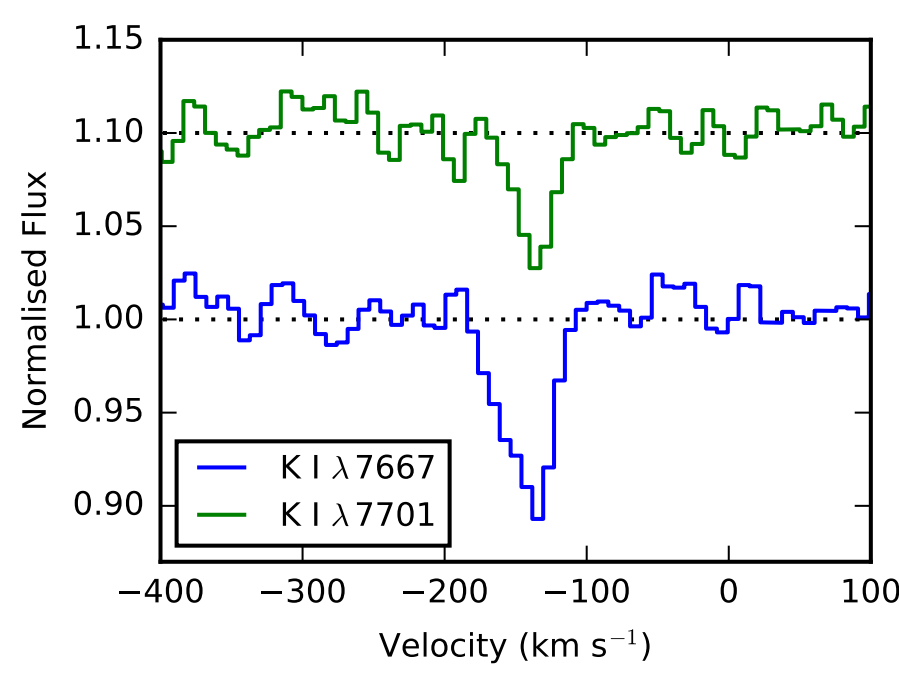

Fig. 3. K I at $\lambda \lambda 7667$ and 7701 from the 14 April X-shooter spectrum. The spectra are normalised and corrected for telluric features.

are strongly blended, it cannot be excluded that there are more unresolved components contributing to the profile.

We further detected K I at $\lambda \lambda 7667$ and 7701 in the X-shooter spectra. Unfortunately, $\mathrm{K}$ I is not covered by UVES with the chosen set-up. In Fig. 3, the K I lines from the spectrum of 14 April is shown, from which we measure $E W \mathrm{~s}=112 \pm 9$ and $71 \pm 6 \mathrm{~m} \AA$, respectively. While the $\mathrm{K} \mathrm{I}$ is also visible in the spectrum from 12 May, several noisy features that do not correspond to telluric lines make precise EW measurements difficult.

We searched all spectra for traces of DIBs as well as $\mathrm{CH}$ and $\mathrm{CH}+$. We found a feature in both UVES and X-shooter spectra which seemingly corresponds to the DIB at $\lambda 6284$ in the host galaxy rest frame. Upon closer inspection however, the feature does not align with the Doppler shift of the other detected absorption features, casting doubt on the identification. Other known DIBs, such as $\lambda \lambda 5780$ and 5797, which are believed to correlate well with reddening (Phillips et al. 2013; Baron et al. 2015), are not detected above the noise level. Assuming fullwidth-half-maxima from SN 2001el for DIBs $\lambda \lambda 5780$ and 5797 of 2.0 and $0.7 \AA$, the corresponding EWs must be $<150$ and $<50 \mathrm{~m} \AA$, respectively, given the signal-to-noise ratio.

From the UV, optical and IR photometry of iPTF16abc we can estimate the reddening of the SN by fitting the SED of SN 2011fe (as in Amanullah et al. 2014, 2015) to the data. Aside from the unusually fast rise of iPTF16abc, the above SED fits the lightcurve and colours well. For this reason, we avoid including the early photometry and the light-curves using the photometry between phases $p=-10-+40$ days.

The measured reddening is estimated to $E(B-V)=$ 0.07 (0.05) mag for $R_{V}=1.4$ (3.1), that is, the full range of $R_{V}=1.4-3.1$ values measured for SNe Ia (e.g. Amanullah et al. 2015). The fit further determined the light-curve parameters $t_{B_{\max }}=57498.76$ and $s=1.074$, which define the $B$-band maximum brightness in MJD and the lightcurve stretch with respect to SN 20011fe (see e.g. Goldhaber et al. 2001, for a discussion on lightcurve stretch), respectively. All statistical uncertainties are subdominant, and the errors are dominated by the assumed value of $R_{V}$. The fitted values of $E(B-V)$ correspond to an extinction of $A_{V}=0.1$ (0.2) mag.

The extinction can also be estimated by taking advantage of the standard candle property of SNe Ia and comparing the measured peak brightness with the expected for the given redshift, $z=0.0234$, of NGC 5221. Using the SN Ia lightcurve fitter SALT2 (Guy et al. 2007), we find that $A_{V}=-0.03 \pm 0.04$ which is well within the expected intrinsic peak brightness dispersion of $0.1 \mathrm{mag}$.

In the past, grey dust ejected from galaxies has been proposed (Aguirre 1999a,b; Goobar et al. 2002) as an alternative explanation to the observations interpreted as a cosmological constant driven acceleration of the universe (Perlmutter et al. 1999; Riess et al. 1998). Although quasar observations have shown that grey dust is not responsible for the dim appearance of cosmological SN Ia samples (Mörtsell \& Goobar 2003; Ménard et al. 2010), a small grey dust component could still lead to biased measurements of cosmological samples. Matter streaming from a galaxy is a suggested location in which grey dust could be forming. In such a scenario the line-of-sight to iPTF16abc should probe an overdensity of grey dust, and the standard candle properties of this SN Ia can be used to constrain it. Since the peak brightness of iPTF16abc is within the intrinsic scatter of SNe Ia however, the grey dust column along the line-of-sight must be negligible.

\section{Absence of circumstellar absorption}

Although the majority of the absorption lines detected in SN Ia spectra are believed to be due to gas in the ISM of their host galaxies, there exist observations that indicate the presence of CS gas. For example, cases of slight variations in absorption line profiles (Patat et al. 2007, 2013; Simon et al. 2009; Graham et al. 2015; Ferretti et al. 2016) hint at photoionisation or recombination of absorbers and predominantly blue-shifted Na I D profiles (Sternberg et al. 2011; Maguire et al. 2013) suggest outflowing gas.

Nevertheless, the existence of CS gas surrounding SNe Ia remains uncertain. Most of the known cases of varying absorption lines all occurred in crowded fields, where geometric effects (Patat et al. 2010) might be expected and a larger sample of multi-epoch high-resolution spectra has not revealed any more examples (Sternberg et al. 2014).

SNe Ia peak in UV earlier than in optical. Because of this, most photoionisation should occur before the SNe reach maximum brightness (Borkowski et al. 2009; Ferretti et al. 2016). Ideally, early spectra (approximately 14 days before maximum) need to be obtained to search for photoionisation of CS gases. The remote location and early discovery of iPTF16abc made it a good candidate SN Ia to search for CS matter by looking for changes in absorption line profiles.

Knowing the SED of SNe Ia as well as the ionisation energy and cross-section of an absorption species, the rate of photoionisation depends on the distance of the gas from the SN. Thus measuring the ionisation rate, is a method to determine the distance of an absorber to the supernova. Thereby the different ionisation energies and cross-sections of different absorption species implies that they are sensitive to photoionisation at different distances.

In the absence of any variations, one can determine the distances at which there was no detectable gas, which would have been been ionised. The exclusion range is defined by two radii. The inner radius is determined by the distances at which all gas is ionised before the first spectrum is taken. The outer radius is defined by the distance at which the amount of ionisation that occurs by the time the last spectrum is taken is negligible.

In Table 4 we present the time series of the measured EWs of $\mathrm{Na} I \mathrm{D}$ and $\mathrm{Ca}$ II H\&K. It can be seen that there is neither significant time variability in the EW measurements, nor are there any 
R. Ferretti et al.: Probing gas and dust in the tidal tail of NGC 5221 with the type Ia supernova iPTF16abc

Table 3. Average Na I D and Ca II H\&K equivalent widths of the features with centroids at -77 and $-51 \mathrm{~km} \mathrm{~s}^{-1}$ measured from the UVES spectra.

\begin{tabular}{ccccccccc}
\hline \hline $\begin{array}{c}v \\
\left(\mathrm{~km} \mathrm{~s}^{-1}\right)\end{array}$ & $\begin{array}{c}\text { Na I D1 } \\
(\mathrm{m} \AA)\end{array}$ & $\begin{array}{c}\text { Na I D2 } \\
(\mathrm{m} \AA)\end{array}$ & $\begin{array}{c}\text { Ratio } \\
(\mathrm{D} 2 / \mathrm{D} 1)\end{array}$ & $\begin{array}{c}N(\mathrm{Na} \text { I }) \\
\left(\times 10^{12} \mathrm{~cm}^{-2}\right)\end{array}$ & $\begin{array}{c}\text { Ca II H } \\
(\mathrm{m} \AA)\end{array}$ & $\begin{array}{c}\text { Ca II K } \\
(\mathrm{m} \AA)\end{array}$ & $\begin{array}{c}\text { Ratio } \\
(\mathrm{K} / \mathrm{H})\end{array}$ & $\begin{array}{c}N(\mathrm{Ca} \text { II }) \\
\left(\times 10^{12} \mathrm{~cm}^{-2}\right)\end{array}$ \\
\hline-77 & $241 \pm 3$ & $279 \pm 3$ & 1.16 & $>2.4$ & $42 \pm 3$ & $73 \pm 3$ & 1.74 & $1.0 \pm 0.1$ \\
-51 & $278 \pm 3$ & $312 \pm 4$ & 1.12 & $>2.8$ & $110 \pm 3$ & $166 \pm 4$ & 1.51 & $3.0 \pm 0.3$ \\
\hline
\end{tabular}

Notes. The equivalent width ratios are indicative of the optical depth of the absorbers. Column density lower limits are given for Na I, which were computed from $\mathrm{Na}$ I D1 assuming the optically thin relation to equivalent width. The Ca II column densities were determined from the five-component Voigt profile fit.

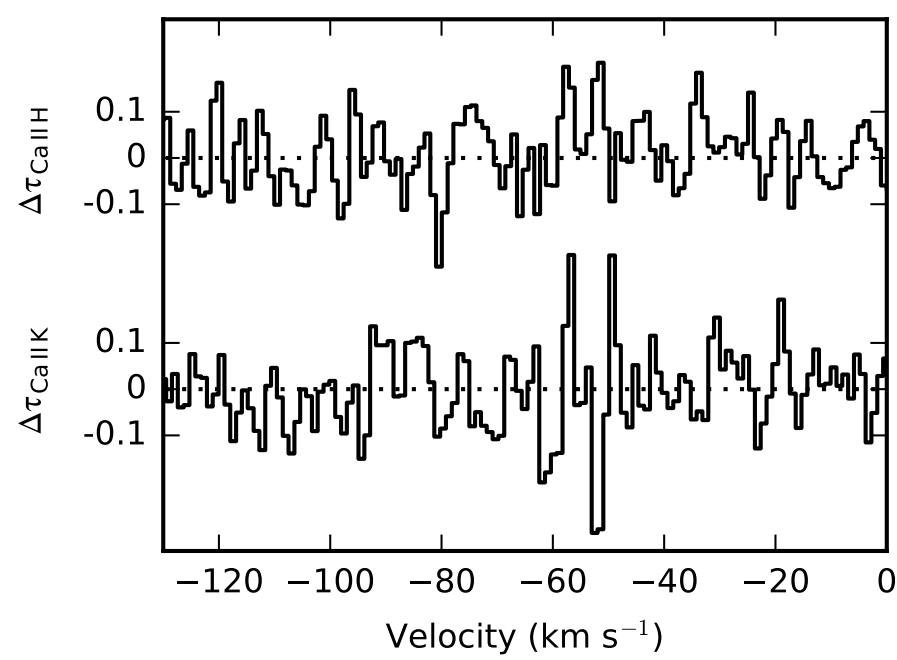

Fig. 4. Difference in apparent optical depth between the UVES spectra of Ca II H\&K. No pixels seem to be significant outliers.

visible changes in the line profiles. We investigate the profiles of $\mathrm{Ca}$ II H\&K in the UVES spectrum in more detail than the saturated $\mathrm{Na} I \mathrm{D}$ lines. A direct comparison of the Voigt profile parameters, reveals no difference between the two UVES epochs. Furthermore, we plot the difference in the apparent optical depth of $\mathrm{Ca}$ II H\&K between the two epochs in Fig. 4. We find no significant changes in the apparent optical depth of any part of the profiles.

Using the method of Ferretti et al. (2016), we can exclude the presence of $\mathrm{Na}$ I gas at a distance of $R_{\mathrm{Na} \mathrm{I}}^{\text {excl }} \approx 1 \times 10^{18}-2 \times 10^{19} \mathrm{~cm}$, and Ca II gas at $R_{\mathrm{Ca} \text { II }}^{\text {excl }} \approx 8 \times 10^{16}-3 \times 10^{17} \mathrm{~cm}$ from iPTF16abc at the $3 \sigma$ confidence level. To illustrate the exclusion range, Fig. 5 shows Na I ionisation curves of gas clouds at the limiting and exclusion radii. The exclusion limits do not include possible errors in the SNe Ia SED as described in Ferretti et al. (2016). In particular, the exclusion limits obtained from Ca II must be taken with caution, due to the diversity of the SN Ia SEDs.

Thus all the detected absorption features must originate from gas farther from iPTF16abc than the outer exclusion limit and are part of the ISM. It is not possible to further constrain the relation of iPTF16abc with the gas clouds other than stating that the supernova must have occurred behind them. Due to the broad nature of intrinsic supernova spectral features, the systemic velocity of the supernova can't be determined with accuracy. It is nevertheless plausible that the progenitor system of iPTF16abc was moving with the tidally stripped gas behind which it exploded.

\section{Discussion}

The position of iPTF16abc suggests that we are probing the ISM in the tidal arm of NGC 5221. iPTF16abc is $170^{\prime \prime}$ from

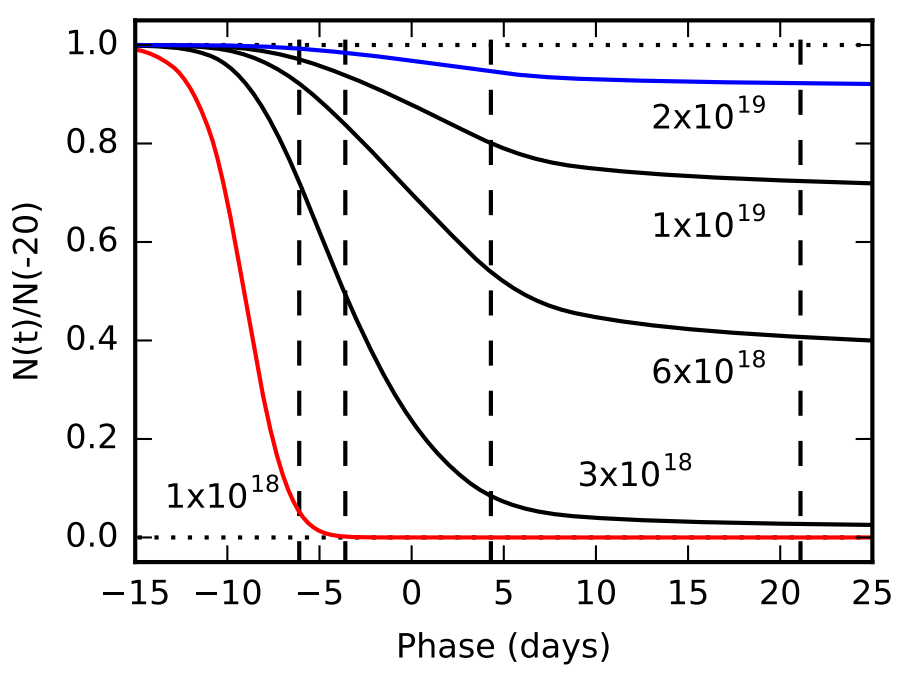

Fig. 5. Fractional photoionisation curves of $\mathrm{Na}$ I gas at annotated radii in $\mathrm{cm}$. The vertical dashed lines indicate the phases of the obtained spectra. The red curve at $1 \times 10^{18} \mathrm{~cm}$, defines the inner exclusion radius at which a gas cloud would have been ionised before the first spectrum was obtained. The blue curve defines the outer exclusion radius at $2 \times 10^{19} \mathrm{~cm}$, where photoionisation leads to negligible change.

the centre of NGC 5221, which at a redshift of $z=0.0234$ (Courtois \& Tully 2015) corresponds to at least $80 \mathrm{kpc}$. The absorption lines span a radial velocity of $-100--30 \mathrm{~km} \mathrm{~s}^{-1}$, with respect to the rest-frame of the host galaxy core. These values are smaller than the rotation velocity of NGC 5221. Courtois \& Tully (2015) quote a line width (twice the rotation velocity) of $510 \pm 20 \mathrm{~km} \mathrm{~s}^{-1}$. Furthermore, two Sloan Digital Sky Survey (SDSS) spectra of spiral arms on opposite sides of the galaxy show a velocity difference of $477 \mathrm{~km} \mathrm{~s}^{-1}$. The coordinates at which the SDSS spectra have been taken prior to explosion, are marked in Fig. 1, whereby the eastern arm (S1) is approaching and the western arm (S2) is receding with -254 and $223 \mathrm{~km} \mathrm{~s}^{-1}$, respectively. Interestingly, this implies that the gas in the tidal tail is receding from the galaxy in the opposite direction to the spiral arm it is closest to. Although the tidal tail projects in the opposite direction, this may indicate that the tidal stream connects to the eastern spiral arm.

To further compare the radial velocity of the gas seen in the iPTF16abc spectra with NGC 5221, we consider an H I profile by the Arecibo $L$-band Feed Array (ALFA) as part of the Arecibo Legacy Fast ALFA survey (ALFALFA, Giovanelli et al. 2005; Haynes et al. 2011). The beam of the ALFA encompasses both the position of iPTF16abc and NGC 5221. In Fig. 6, the Na I D1 profile of the iPTF16abc line-of-sight is compared to the ALFA $\mathrm{H}$ I profile of the entire galaxy.

The position of iPTF16abc allows us to estimate a timeframe within which the gas must have been stripped from NGC 5221. Assuming the gas is moving away from the galaxy 
Table 4. Equivalent widths of Na I D, Ca II H\&K, of iPTF 16abc using X-shooter and UVES.

\begin{tabular}{|c|c|c|c|c|c|c|c|c|}
\hline Instrument & Date & $\begin{array}{l}\text { Phase } \\
\text { (days) }\end{array}$ & $\bar{R}(\lambda / \delta \lambda)$ & $S / N$ & $\begin{array}{c}\text { Na I D1 } \\
(\mathrm{mA})\end{array}$ & $\begin{array}{c}\text { Na I D2 } \\
(\mathrm{mA})\end{array}$ & $\begin{array}{c}\text { Ca II H } \\
(\mathrm{mA})\end{array}$ & $\begin{array}{c}\text { Ca II K } \\
(\mathrm{m \AA})\end{array}$ \\
\hline X-shooter & Apr. 14 & -6.1 & 7450 & 113 & $512 \pm 6$ & $606 \pm 6$ & $141 \pm 4$ & $238 \pm 8$ \\
\hline UVES & Apr. 16 & -3.6 & 68000 & 20 & $517 \pm 7$ & $585 \pm 7$ & $147 \pm 7$ & $240 \pm 6$ \\
\hline UVES & Apr. 24 & 4.3 & 87000 & 25 & $511 \pm 5$ & $597 \pm 5$ & $155 \pm 6$ & $238 \pm 6$ \\
\hline X-shooter & May 12 & 21.2 & 7450 & 73 & $497 \pm 10$ & $592 \pm 10$ & $138 \pm 8$ & $227 \pm 9$ \\
\hline & \multicolumn{4}{|c|}{ Average } & $511 \pm 3$ & $597 \pm 3$ & $145 \pm 3$ & $237 \pm 3$ \\
\hline
\end{tabular}

Notes. The resolution of the UVES spectra were estimated from the full width at half-maximum intensity (FWHM) of several telluric features, while the nominal resolution of the X-shooter spectra are given. The $\mathrm{S} / \mathrm{N}$ per pixel is measured around the wavelength of Na I D.

Table 5. Neighbouring galaxies of NGC 5221 at comparable redshift.

\begin{tabular}{ccccl}
\hline \hline Galaxy & Redshift & $\begin{array}{c}\text { Perp. Dist. } \\
(\mathrm{kpc})\end{array}$ & Direction $^{\dagger}$ & Morphology and/or notes \\
\hline 2MASX J13341283+1349334 & 0.0235 & 296 & $\mathrm{~W}$ & dwarf \\
2MASX J13343006+1348154 & 0.0242 & 184 & WSW & ? \\
SDSS J133443.53+134507.3 & 0.0253 & 161 & $\mathrm{SW}$ & ? elongated dwarf \\
2MASX J13344815+1344391 & 0.0222 & 159 & SSW & dwarf \\
NGC 5222 & 0.0231 & 154 & $\mathrm{~S}$ & large elliptical \\
- & 0.0228 & 150 & $\mathrm{~S}$ & irregular overlapping merging with NGC 5222 \\
NGC 5230 & 0.0229 & 363 & SE & large face-on spiral \\
SDSS J133509.69+135324.0 & 0.0234 & 136 & NE & dwarf \\
NGC 5226 & 0.0244 & 161 & NNE & spiral? \\
\hline
\end{tabular}

Notes. ${ }^{(\dagger)}$ At $z=0.0234$.

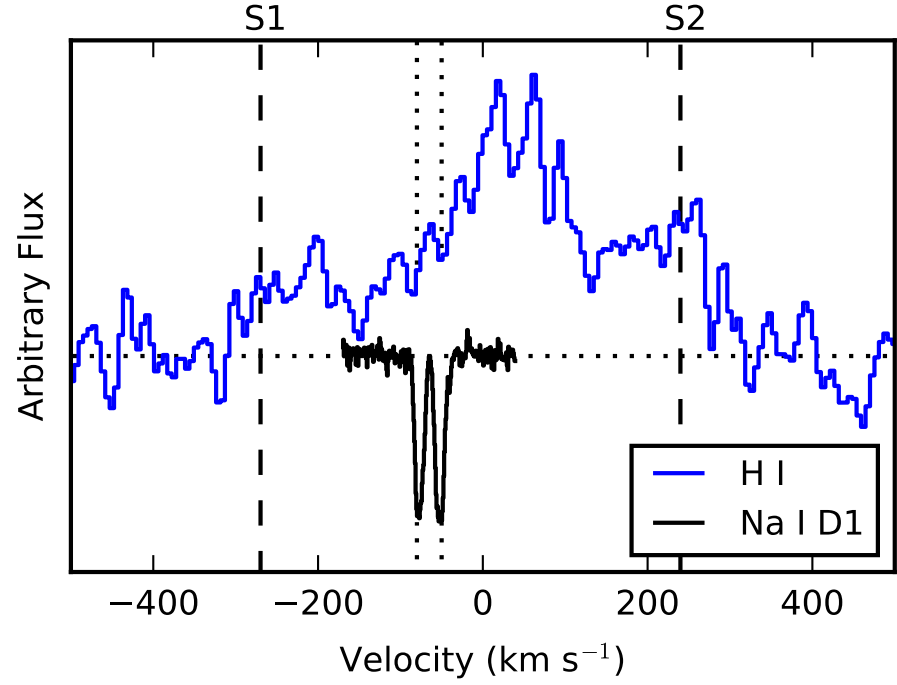

Fig. 6. Comparison of the radial velocity of the $\mathrm{Na} I$ gas along the lineof-sight of iPTF16abc and H I in and around NGC 5221. The ALFA $\mathrm{H}$ I spectrum encompasses the position of iPTF16abc and NGC 5221. The Na I D1 profile is shown from the Apr. 24 UVES spectrum. Vertical dashed lines indicate the line-of-sight velocity of the two SDSS spectra with respect to the rest-frame of NGC 5221. The labels S1 and S2 corresponding to those in Fig. 1.

with a velocity of $v \approx 100 \mathrm{~km} \mathrm{~s}^{-1}$, it would take at least $8 \times 10^{8} \mathrm{yr}$ to reach a distance of $80 \mathrm{kpc}$, depending on the exact projection angle. Models show that this time frame is consistent with close encounters and mergers of large galaxies (Toomre \& Toomre 1972).

A number of galaxies, listed in Table 5, are at a comparable redshift to NGC 5221 and within a few hundred kpc from it. The two most prominent galaxies in the list,
NGC 5222 and NGC 5230, are likely candidates to have interacted with NGC 5221, producing the observed tidal stream. Hallenbeck et al. (2016) have studied NGC 5230 as an example of a very $\mathrm{H}$ I rich galaxy. In fact, the ALFALFA data they present in their study show that H I gas encompasses the entire galaxy group, with column density maxima corresponding to NGC 5221, NGC 5222 and NGC 5230. Thus the gas along the line-of-sight of iPTF16abc is representative of gas that can end up in the CGM of the group via interactions between the galaxies.

Narrow absorption lines, such as those detected in the spectra of iPTF16abc, are not unusual features for SN Ia spectra (e.g. the sample of Sternberg et al. 2011). However, these features typically appear in lines-of-sight of supernovae clearly situated within their host galaxies. The $\mathrm{Na}$ I and $\mathrm{Ca}$ II gas columns along the line-of-sight of iPTF16abc appear to be comparable to typical lines-of-sight within galaxies. To illustrate this, we consider the $\mathrm{Na} I \mathrm{D}$ absorption in the two SDSS spectra available of NGC 5221. We find Na I D EWs of $2.1 \pm 0.5$ and $2.9 \pm 0.9 \AA$ for the eastern and western spectra respectively. Ca II H\&K lines are unfortunately buried in noise in the SDSS spectra and cannot be measured.

Although the $\mathrm{Na}$ I columns in the tidal stream and the NGC 5221 differ by close to an order of magnitude, it is important to note the differences between the SDSS and supernova $\mathrm{Na} I \mathrm{D}$ EWs. In the case of the SDSS spectra, the absorption lines represent the average absorption along the lines-of-sight to all stars covered by the spectral fibre. The investigated column can thus be considered to be the average across an area of $\approx 6 \mathrm{kpc}^{2}$ area of the galaxy. In comparison with this, the column measured in the supernova spectrum, spans the projected area of a photosphere which is of the order of $\approx 10^{-6} \mathrm{pc}^{2}$ at maximum brightness ${ }^{2}$. We are thus comparing a very small area in the tidal

\footnotetext{
2 Approximated from $10^{4} \mathrm{~km} \mathrm{~s}^{-1}$ expansion after 21 days.
} 
tail to the average of a large area within the galaxy, where many more gas clouds with different velocities can be situated along the line-of-sight.

From ISM studies of the Milky Way, it is known that Na I D and $\mathrm{Ca}$ II H\&K correlate with reddening and also $\mathrm{H}$ I column densities (Poznanski et al. 2012; Murga et al. 2015). These empirical relations can be used to estimate $E(B-V)$ and $N(\mathrm{H} \mathrm{I})$ for the line-of-sight of iPTF16abc. Using the saturated Na I D lines, the Poznanski et al. (2012) relation suggests that $E(B-$ $V)_{\text {est }}>0.32$. It is known that the empirical relations of $\mathrm{Na}$ I D to reddening work poorly for SNe Ia (Poznanski et al. 2011; Phillips et al. 2013). iPTF16abc is another example with deep $\mathrm{Na}$ I D absorption lines, but comparatively little photometric reddening. Recently, Hoang (2017) suggested that the unusually large Na I columns could originate from gas released during dust grain collisions in clouds irradiated by the SNe. The ratio $N(\mathrm{Na} \mathrm{I}) / N(\mathrm{Ca} \mathrm{II})>1.3$ falls outside the range over which the Murga et al. (2015) relation to H I has been determined. Extrapolating the relation would imply an $\mathrm{H} \mathrm{I}$ column $N(\mathrm{H} \mathrm{I})_{\text {est. }}>$ $10^{21} \mathrm{~cm}^{-2}$.

The H I column density estimates are more difficult to compare with. From the ALFA spectrum, we can compute that the entire beam, spanning $3.5^{\prime} \times 3.8^{\prime}$ contains $M_{\mathrm{H} \mathrm{I}}=1.0 \times 10^{10} M_{\odot}$, corresponding to an average column density of $N(\mathrm{H} \mathrm{I})=3.7 \times$ $10^{19} \mathrm{~cm}^{-2}$. Most of the H I in the ALFA spectrum must be situated in NGC 5221, but could also have been stripped to locations such as the line-of-sight of iPTF16abc.

\section{Summary and conclusions}

We have presented the interstellar absorption lines observed in the spectra of Type Ia supernova iPTF16abc. The gas corresponds to the tidally stripped ISM at least $80 \mathrm{kpc}$ from the centre of the host galaxy NGC 5221. Compared with a line-of-sight in a typical galaxy, the ISM in the tidal tail appears to have a typical gas content, but a surprisingly small column of dust.

We detected $\mathrm{Na}$ I D and Ca II H\&K absorption features in two distinct clusters at velocities -77 and $-51 \mathrm{~km} \mathrm{~s}^{-1}$ from the systemic velocity of NGC 5221.

From the lack of variations in the Na I D and Ca II H\&K profiles, we determined that the observed gas cannot be part of the CS environment of the supernova, since photoionisation would have resulted in a significant change in the column density. Thus the gas seen along the line-of-sight of iPTF16abc is the tidally stripped ISM of NGC 5221.

At the same time the standard candle nature of iPTF16abc has allowed us to determine that the supernova is barely reddened compared with the normal SNe Ia colours, implying that there is a negligible amount of dust along the line-of-sight. This further excludes the presence of grey dust, which could be along the line-of-sight of iPTF16abc, if grey dust existed in the intergalactic medium.

NGC 5221 likely had a close encounter with one of its neighbouring galaxies in the past $\approx 10^{9} \mathrm{yr}$, when large portions of gas and the progenitor system of iPTF16abc were tidally stripped from it. While the velocity of the gas in the tidal arm appears comparable to the velocity of the eastern spiral arm, the projection of the tail points in the opposite direction. This suggests that the tidal tail might be connected to the eastern spiral arm.

A map of H I content of the group of galaxies including NGC 5221, presented by Hallenbeck et al. (2016), suggests that the group shares a common gas envelope. As the galaxies within the group are likely to continue to interact and merge in the future, the gas seen along the line-of-sight of iPTF16abc is representative of the gas that is transplanted into the CGM surrounding the galaxy group. It has been shown that dense cold gas clouds can exist in the CGM of galaxy groups for $>5 \times 10^{8} \mathrm{yr}$ (Borthakur et al. 2015). In future interactions with neighbouring galaxies, these clouds could be the sites of star formation. In the long run however, the gas clouds could dissipate, enriching the CGM with the detected metals.

The presented gas profiles along the line-of-sight of iPTF16abc should be useful to future studies of NGC 5221, its tidal tail and the galaxy group it is located in. In particular, the profiles could complement higher resolution observations of the $\mathrm{H}$ I gas in NGC 5221 and the tidal arm, resolving the dynamics of the galaxy group.

Acknowledgements. We thank Gregory Hallenbeck for his help with extracting ALFALFA data, David Martinez-Delgado, Darach Watson and Brice Menard for their helpful insights and Jesper Sollerman and Avishay Gal-Yam for their comments. R.A. and A.G. acknowledge support from the Swedish Research Council and the Swedish Space Board. The Oskar Klein Centre is funded by the Swedish Research Council. This work is based on observations made with the Nordic Optical Telescope, operated by the Nordic Optical Telescope Scientific Association at the Observatorio del Roque de los Muchachos, La Palma, Spain, of the Instituto de Astrofisica de Canarias. This work makes use of observations from the LCOGT network. The Pan-STARRS1 Surveys (PS1) and the PS1 public science archive have been made possible through contributions by the Institute for Astronomy, the University of Hawaii, the Pan-STARRS Project Office, the MaxPlanck Society and its participating institutes, the Max Planck Institute for Astronomy, Heidelberg and the Max Planck Institute for Extraterrestrial Physics, Garching, The Johns Hopkins University, Durham University, the University of Edinburgh, the Queen's University Belfast, the Harvard-Smithsonian Center for Astrophysics, the Las Cumbres Observatory Global Telescope Network Incorporated, the National Central University of Taiwan, the Space Telescope Science Institute, the National Aeronautics and Space Administration under Grant No. NNX08AR22G issued through the Planetary Science Division of the NASA Science Mission Directorate, the National Science Foundation Grant No. AST1238877, the University of Maryland, Eotvos Lorand University (ELTE), the Los Alamos National Laboratory, and the Gordon and Betty Moore Foundation.

\section{References}

Aguirre, A. 1999a, ApJ, 525, 583

Aguirre, A. N. 1999b, ApJ, 512, L19

Amanullah, R., Goobar, A., Johansson, J., et al. 2014, ApJ, 788, L21

Amanullah, R., Johansson, J., Goobar, A., et al. 2015, MNRAS, 453, 3300

Baron, D., Poznanski, D., Watson, D., Yao, Y., \& Prochaska, J. X. 2015, MNRAS, 447, 545

Borkowski, K. J., Blondin, J. M., \& Reynolds, S. P. 2009, ApJ, 699, L64

Borthakur, S., Yun, M. S., Verdes-Montenegro, L., et al. 2015, ApJ, 812, 78

Cenko, S. B., Cao, Y., Kasliwal, M., et al. 2016, ATel, 8909

Courtois, H. M., \& Tully, R. B. 2015, MNRAS, 447, 1531

Dekker, H., D’Odorico, S., Kaufer, A., Delabre, B., \& Kotzlowski, H. 2000, in Optical and IR Telescope Instrumentation and Detectors, eds. M. Iye, \& A. F. Moorwood, SPIE Conf. Ser., 4008, 534

Drake, A. J., Djorgovski, S. G., Graham, M. J., et al. 2013a, Central Bureau Electronic Telegrams, 3555

Drake, A. J., Djorgovski, S. G., Mahabal, A. A., et al. 2013b, ATel, 5097 Ferretti, R., Amanullah, R., Goobar, A., et al. 2016, A\&A, 592, A40 Flewelling, H. A., Magnier, E. A., Chambers, K. C., et al. 2016, ArXiv e-prints [arXiv: 1612.05243]

Foley, R. J., Fox, O. D., McCully, C., et al. 2014, MNRAS, 443, 2887

Fox, O. D., Kutyrev, A. S., Rapchun, D. A., et al. 2012, in High Energy, Optical, and Infrared Detectors for Astronomy V, Proc. SPIE, 8453, 845310 Gehrels, N., Chincarini, G., Giommi, P., et al. 2004, ApJ, 611, 1005 Giovanelli, R., Haynes, M. P., Kent, B. R., et al. 2005, AJ, 130, 2598 Goldhaber, G., Groom, D. E., Kim, A., et al. 2001, ApJ, 558, 359 Goobar, A., \& Leibundgut, B. 2011, Ann. Rev. Nucl. Part. Sci., 61, 251 Goobar, A., Bergström, L., \& Mörtsell, E. 2002, A\&A, 384, 1 Goobar, A., Johansson, J., Amanullah, R., et al. 2014, ApJ, 784, L12 Graham, M. L., Valenti, S., Fulton, B. J., et al. 2015, ApJ, 801, 136 Guy, J., Astier, P., Baumont, S., et al. 2007, A\&A, 466, 11

Hallenbeck, G., Huang, S., Spekkens, K., et al. 2016, AJ, 152, 225 Haynes, M. P., Giovanelli, R., Martin, A. M., et al. 2011, AJ, 142, 170 
Hoang, T. 2017, ApJ, 836, 13

Kausch, W., Noll, S., Smette, A., et al. 2015, A\&A, 576, A78

Maguire, K., Sullivan, M., Thomas, R. C., et al. 2011, MNRAS, 418, 747

Maguire, K., Sullivan, M., Patat, F., et al. 2013, MNRAS, 436, 222

Maoz, D., Mannucci, F., \& Brandt, T. D. 2012, MNRAS, 426, 3282

Ménard, B., Kilbinger, M., \& Scranton, R. 2010, MNRAS, 406, 1815

Miller, A. A., Laher, R., Masci, F., et al. 2016, ATel, 8907

Miller, A. A., Cao, Y., Piro, A. L., et al. 2017, ApJ, submitted, ArXiv e-prints [arXiv: 1708.07124]

Modigliani, A., Goldoni, P., Royer, F., et al. 2010, in Observatory Operations: Strategies, Processes, and Systems III, Proc. SPIE, 7737, 773728

Mörtsell, E., \& Goobar, A. 2003, J. Cosmol. Astropart. Phys., 9, 009

Murga, M., Zhu, G., Ménard, B., \& Lan, T.-W. 2015, MNRAS, 452, 511

Patat, F., Chandra, P., Chevalier, R., et al. 2007, Science, 317, 924

Patat, F., Cox, N. L. J., Parrent, J., \& Branch, D. 2010, A\&A, 514, A78

Patat, F., Cordiner, M. A., Cox, N. L. J., et al. 2013, A\&A, 549, A62

Perlmutter, S., Aldering, G., Goldhaber, G., et al. 1999, ApJ, 517, 565

Phillips, M. M., Simon, J. D., Morrell, N., et al. 2013, ApJ, 779, 38

Poznanski, D., Ganeshalingam, M., Silverman, J. M., \& Filippenko, A. V. 2011, MNRAS, 415, L81
Poznanski, D., Prochaska, J. X., \& Bloom, J. S. 2012, MNRAS, 426, 1465 Riess, A. G., Filippenko, A. V., Challis, P., et al. 1998, AJ, 116, 1009 Ritchey, A. M., Welty, D. E., Dahlstrom, J. A., \& York, D. G. 2015, ApJ, 799, 197

Roming, P. W. A., Kennedy, T. E., Mason, K. O., et al. 2005, Space Sci. Rev., 120,95

Schlafly, E. F., \& Finkbeiner, D. P. 2011, ApJ, 737, 103

Schombert, J. M., Wallin, J. F., \& Struck-Marcell, C. 1990, AJ, 99, 497

Simon, J. D., Gal-Yam, A., Gnat, O., et al. 2009, ApJ, 702, 1157

Smette, A., Sana, H., Noll, S., et al. 2015, A\&A, 576, A77

Snow, T. P. 2001, Spectrochimica Acta, 57, 615

Snow, T. P., \& McCall, B. J. 2006, ARA\&A, 44, 367

Sollerman, J., Cox, N., Mattila, S., et al. 2005, A\&A, 429, 559

Sternberg, A., Gal-Yam, A., Simon, J. D., et al. 2011, Science, 333, 856

Sternberg, A., Gal-Yam, A., Simon, J. D., et al. 2014, MNRAS, 443, 1849

Toomre, A., \& Toomre, J. 1972, ApJ, 178, 623

Vernet, J., Dekker, H., D’Odorico, S., et al. 2011, A\&A, 536, A105

Welty, D. E., Ritchey, A. M., Dahlstrom, J. A., \& York, D. G. 2014, ApJ, 792, 106

Yang, Y., Wang, L., Baade, D., et al. 2017, ApJ, 834, 60 